\title{
Irrationalité de la somme des inverses de la suite de Fibonacci
}

Daniel Duverney

Daniel Duverney est né en 1955 à Montpellier (France). Il est entré à l'Ecole Normale Supérieure de Cachan en 1974 et a obtenu l'Agrégation de Mathématiques en 1977. Après avoir enseigné en France dans différents lycées (Lille, Bourges), il a obtenu son Doctorat de Mathématiques en 1993 et son Habilitation à diriger des recherches en 1996. Ses recherches portent sur la théorie des nombres, plus précisément sur les problèmes d'irrationalité et de transcendance. Actuellement, il est Professeur de Mathématiques Spéciales au Lycée Technique Baggio à Lille.

Soit $\left(F_{n}\right)$ la suite de Fibonacci, définie par $F_{0}=0, F_{1}=1$ et la relation de récurrence

$$
F_{n+1}=F_{n}+F_{n-1} .
$$

On sait que

$$
F_{n}=\frac{\Phi^{n}-\Psi^{n}}{\Phi-\Psi},
$$

où $\Phi=(1+\sqrt{5}) / 2$ est le nombre d'or (voir par exemple [4]), et $\Psi=-1 / \Phi$.

Le but de cet article est de donner une démonstration simple de l'irrationalité de la somme

$$
S=\sum_{n=1}^{+\infty} \frac{1}{F_{n}} .
$$

L'irrationalité de $S$ a été démontrée pour la première fois par R. André-Jeannin en 1989 [1]. La démonstration proposée ici s'inspire, en la simplifiant, de celle donnée par P. Bundschuh et K. Vâánánen dans un article paru en 1994 [3]. Elle est fondée

Die Folge der Fibonacci-Zahlen dürfte eine der bestuntersuchten der ganzen mathematischen Welt sein. Trotzdem gibt sie immer wieder Anlass zu neuen Überlegungen und Überraschungen. Dabei kommen oft — wie zum Beispiel im vorliegenden Beitrag von Daniel Duverney - neuartige Methoden zum Tragen, die an ganz anderen Fragestellungen entwickelt worden sind. ust 
sur certaines propriétés des fonctions q-exponentielle et q-logarithme, qui se définissent comme suit; on pose, pour $|q|>1$ :

$$
\begin{aligned}
& \operatorname{Exp}_{q} x=1+\sum_{n=1}^{+\infty} \frac{x^{n}}{(q-1)\left(q^{2}-1\right) \ldots\left(q^{n}-1\right)} \quad(x \in \mathbb{C}) \\
& \log _{q} x=\sum_{n=1}^{+\infty} \frac{x^{n}}{q^{n}-1} \quad(|x|<|q|) .
\end{aligned}
$$

Les fonctions $\operatorname{Exp}_{q}$ et $\log _{q}$ sont considérées comme des q-analogues [5] des fonctions exponentielles et logarithmes ordinaires, car on peut vérifier que $\lim _{q \rightarrow 1} \operatorname{Exp}_{q}[(q-1) x]=e^{x}$ et $\lim _{q \rightarrow 1}(q-1) \log _{q} x=-\ln (1-x)$.

Nous aurons besoin des trois lemmes suivants:

Lemme 1. $\operatorname{Exp}_{q} x=\prod_{n=1}^{+\infty}\left(1+\frac{x}{q^{n}}\right), \forall x \in \mathbb{C}$.

Démonstration. On vérifie facilement, à partir de (4), que la fonction $q$-exponentielle vérifie

$$
\operatorname{Exp}_{q} x=\left(1+\frac{x}{q}\right) \operatorname{Exp}_{q}\left(\frac{x}{q}\right)
$$

Par récurrence, il vient facilement $\forall n \in \mathbb{N}$

$$
\operatorname{Exp}_{q} x=\left(1+\frac{x}{q}\right)\left(1+\frac{x}{q^{2}}\right) \ldots\left(1+\frac{x}{q^{n}}\right) \operatorname{Exp}_{q}\left(\frac{x}{q^{n}}\right)
$$

Lorsque $n \rightarrow+\infty, \operatorname{Exp}_{q}\left(\frac{x}{q^{n}}\right) \rightarrow 1$, et le lemme 1 en résulte.

Lemme 2. $\log _{q} x=\sum_{n=1}^{\infty} \frac{x}{q^{n}-x},|x|<1$.

Démonstration. On peut vérifier, à partir de (5), que

$$
\log _{q} x=\log _{q}\left(\frac{x}{q}\right)+\frac{x}{q-x}
$$

On en déduit $\forall n \in \mathbb{N}$

$$
\log _{q} x=\log _{q}\left(\frac{x}{q^{n}}\right)+\sum_{k=1}^{n} \frac{x}{q^{k}-x}
$$

On fait tendre $n$ vers $+\infty$, et le lemme 2 s'en déduit. 
Lemme 3. On suppose que $x$ et $q$ sont réels, avec $|q|>1$ et $|x|<|q|$. Alors

$$
\log _{q} x=x \frac{\left(\operatorname{Exp}_{q}\right)^{\prime}(-x)}{\operatorname{Exp}_{q}(-x)}
$$

Démonstration. On prend le logarithme (ordinaire) de (7)

$$
\ln \left(\operatorname{Exp}_{q}(-x)\right)=\sum_{k=1}^{n}\left(1-\frac{x}{q^{k}}\right)+\ln \left(\operatorname{Exp}_{q}\left(-\frac{x}{q^{n}}\right)\right) .
$$

On dérive

$$
-\frac{\left(\operatorname{Exp}_{q}\right)^{\prime}(-x)}{\operatorname{Exp}_{q}(-x)}=-\sum_{k=1}^{n} \frac{1}{q^{k}-x}+\frac{\left(\operatorname{Exp}_{q}\right)^{\prime}\left(-\frac{x}{q^{n}}\right)}{\operatorname{Exp}_{q}\left(-\frac{x}{q^{n}}\right)} \cdot \frac{-1}{q^{n}}
$$

On a $\lim _{n \rightarrow+\infty}\left(\operatorname{Exp}_{q}\right)^{\prime}\left(-\frac{x}{q^{n}}\right)=\frac{1}{q-1}, \lim _{n \rightarrow+\infty} \operatorname{Exp}_{q}\left(-\frac{x}{q^{n}}\right)=1$.

D'où

$$
\frac{\left(\operatorname{Exp}_{q}\right)^{\prime}(-x)}{\operatorname{Exp}_{q}(-x)}=\sum_{k=1}^{+\infty} \frac{1}{q^{k}-x} .
$$

Le lemme 3 résulte donc du lemme 2.

Démontrons maintenant l'irrationalité de la somme $S=\sum_{n=1}^{+\infty} \frac{1}{F_{n}}$. Dans l'expression (5) du $q$-logarithme, nous prenons $q=-\Phi^{2}$ et $x=-\Phi$. Il vient

$$
\log _{-\Phi^{2}}(-\Phi)=\sum_{n=1}^{+\infty} \frac{(-\Phi)^{n}}{\left(-\Phi^{2}\right)^{n}-1}=\sum_{n=1}^{+\infty} \frac{1}{\Phi^{n}-\Psi^{n}}
$$

d'où

$$
S=\sum_{n=1}^{+\infty} \frac{1}{F_{n}}=(\Phi-\Psi) \log _{-\Phi^{2}}(-\Phi) .
$$

Supposons que $S=A / B,(A, B) \in \mathbb{Z} \times(\mathbb{Z}-\{0\})$.

On a alors $-B(\Phi-\Psi) \log _{-\Phi^{2}}(-\Phi)+A=0$, et on déduit du lemme 3 que

$$
B(\Phi-\Psi) \Phi\left(\operatorname{Exp}_{-\Phi^{2}}\right)^{\prime}(\Phi)+A \cdot \operatorname{Exp}_{-\Phi^{2}}(\Phi)=0 .
$$

Si on remplace les $q$-exponentielles par leur expression sous forme de séries déduites de (4), il vient

$$
A+\sum_{n=1}^{+\infty} \frac{A+B n(\Phi-\Psi)}{\left(1+\Phi^{2}\right)\left(1-\Phi^{4}\right) \ldots\left(1-\left(-\Phi^{2}\right)^{n}\right)}(-\Phi)^{n}=0
$$


Soit $N \in \mathbb{N}-\{0\}$; dans (12) nous séparons la somme en deux:

$$
\begin{aligned}
A & +\sum_{n=1}^{N} \frac{[A+B n(\Phi-\Psi)](-\Phi)^{n}}{\left(1+\Phi^{2}\right)\left(1-\Phi^{4}\right) \ldots\left(1-\left(-\Phi^{2}\right)^{n}\right)}= \\
& -\sum_{n=N+1}^{+\infty} \frac{[A+B n(\Phi-\Psi)](-\Phi)^{n}}{\left(1+\Phi^{2}\right)\left(1-\Phi^{4}\right) \ldots\left(1-\left(-\Phi^{2}\right)^{n}\right)} .
\end{aligned}
$$

On multiplie le tout $\operatorname{par}\left(1+\Phi^{2}\right)\left(1-\Phi^{4}\right) \ldots\left(1-\left(-\Phi^{2}\right)^{N}\right)$, et on majore en valeur absolue:

$$
\begin{aligned}
& \mid A\left(1+\Phi^{2}\right)\left(1-\Phi^{4}\right) \ldots\left(1-\left(-\Phi^{2}\right)^{N}\right) \\
& \quad+\sum_{n=1}^{N}[A+B n(\Phi-\Psi)](-\Phi)^{n}\left(1-\left(-\Phi^{2}\right)^{n+1}\right) \ldots\left(1-\left(-\Phi^{2}\right)^{N}\right) \mid \\
& \quad \leq \sum_{n=N+1}^{+\infty}|A+B n(\Phi-\Psi)| \frac{\Phi^{n}}{\left(\Phi^{2 N+2}-1\right) \ldots\left(\Phi^{2 n}-1\right)} .
\end{aligned}
$$

Notons $R_{N}$ la somme qui figure dans la partie droite de (14). On a pour $N$ assez grand

$$
\begin{aligned}
& R_{N} \leq \sum_{n=N+1}^{+\infty} N^{2}(n-N) \frac{\Phi^{n}}{\left(\Phi^{2}-\frac{1}{2}\right)^{N+1} \ldots\left(\Phi^{2}-\frac{1}{2}\right)^{n}} \\
& R_{N} \leq N^{2} \sum_{n=N+1}^{+\infty}(n-N)\left(\frac{2 \Phi}{2 \Phi+1}\right)^{n} \\
& R_{N} \leq N^{2}\left(\frac{2 \Phi}{2 \Phi+1}\right)^{N} \sum_{n=N+1}^{+\infty}(n-N)\left(\frac{2 \Phi}{2 \Phi+1}\right)^{n-N}
\end{aligned}
$$

d'où

$$
R_{N} \leq N^{2}\left(\frac{2 \Phi}{2 \Phi+1}\right)^{N} \sum_{m=1}^{+\infty} m\left(\frac{2 \Phi}{2 \Phi+1}\right)^{m}
$$

Soit $X_{N}$ le nombre dont la valeur absolue figure dans la partie gauche de (14). Posons

$$
\begin{aligned}
\widetilde{X_{N}}= & A\left(1+\Psi^{2}\right)\left(1-\Psi^{4}\right) \ldots\left(1-\left(-\Psi^{2}\right)^{N}\right) \\
& +\sum_{n=1}^{N}[A+B n(\Psi-\Phi)](-\Psi)^{n}\left(1-\left(-\Psi^{2}\right)^{n+1}\right) \ldots\left(1-\left(-\Psi^{2}\right)^{N}\right) .
\end{aligned}
$$

Le nombre $\widetilde{X_{N}}$ a été obtenu à partir de $X_{N}$ en échangeant les nombres $\Phi=(1+\sqrt{5}) / 2$ et $\Psi=(1-\sqrt{5}) / 2$. Il en résulte que $X_{N}$ est de la forme $\alpha_{N}+\beta_{N} \sqrt{5}$, avec $\left(\alpha_{N}, \beta_{N}\right) \in \mathbb{Q}^{2}$, 
tandis que $\widetilde{X_{N}}$ est de la forme $\alpha_{N}-\beta_{N} \sqrt{5}$. Par ailleurs, l'expression $X_{N} \widetilde{X_{N}}$ est un polynôme à coefficients entiers, symétrique en $\Phi$ et $\Psi$. Elle s'exprime donc comme un polynôme à coefficients entiers des variables $\Phi+\Psi=1$ et $\Phi \Psi=-1$ ([2], pages 161-163). Donc

$$
X_{N} \widetilde{X_{N}} \in \mathbb{Z}
$$

De plus, $\left|\widetilde{X_{N}}\right|$ se majore facilement. On a en effet

$$
\left|\widetilde{X_{N}}\right| \leq|A| \prod_{k=1}^{N}\left(1+\left|\Psi^{2}\right|^{k}\right)+\sum_{n=1}^{N}(|A|+|B| n(\Phi-\Psi)) \prod_{k=n+1}^{N}\left(1+\left|\Psi^{2}\right|^{k}\right) .
$$

Or on sait (lemme 1) que le produit infini $\prod_{k=1}^{+\infty}\left(1+\left|\Psi^{2}\right|^{k}\right)$ converge vers $\operatorname{Exp}_{\left|\Psi^{-2}\right|}(1)$. Il résulte donc de (18) qu'il existe une constante positive $C$ telle que, pour $N$ assez grand,

$$
\left|\widetilde{X_{N}}\right| \leq C N^{2}
$$

Maintenant, nous multiplions l'inégalité (14) par $\left|\widetilde{X_{N}}\right|$.

En tenant compte de (15) et (19), on voit qu'il existe une constante positive $D$ telle que, pour $N$ assez grand,

$$
\left|X_{N} \widetilde{X_{N}}\right| \leq D N^{4}\left(\frac{2 \Phi}{2 \Phi+1}\right)^{N}
$$

D'où $\lim _{N \rightarrow+\infty}\left|X_{N} \widetilde{X_{N}}\right|=0$. Mais $X_{N} \widetilde{X_{N}} \in \mathbb{Z}$. Donc, pour $N$ assez grand, $X_{N} \widetilde{X_{N}}=0$. Or on a vu que $X_{N}=\alpha_{N}+\beta_{N} \sqrt{5}$ et $\widetilde{X_{N}}=\alpha_{N}-\beta_{N} \sqrt{5}$; pour $N$ assez grand, l'un au moins des deux nombres $X_{N}$ ou $\widetilde{X_{N}}$ est nul, donc $\alpha_{N}=\beta_{N}=0$ car $\sqrt{5}$ est irrationnel. Ainsi $X_{N}=0$ pour $N$ assez grand. Donc $Y_{N}=X_{N} /\left(1+\Phi^{2}\right)\left(1-\Phi^{4}\right) \ldots\left(1-\left(-\Phi^{2}\right)^{N}\right)$ est nul pour $N$ assez grand. Or

$$
\Upsilon_{N}=A+\sum_{n=1}^{N} \frac{[A+B n(\Phi-\Psi)](-\Phi)^{n}}{\left(1+\Phi^{2}\right)\left(1-\Phi^{4}\right) \ldots\left(1-\left(-\Phi^{2}\right)^{n}\right)}
$$

(voir le passage de (13) à (14)). Donc

$$
\Upsilon_{N}-\Upsilon_{N-1}=\frac{[A+B N(\Phi-\Psi)](-\Phi)^{N}}{\left(1+\Phi^{2}\right)\left(1-\Phi^{4}\right) \ldots\left(1-\left(-\Phi^{2}\right)^{N}\right)}=0
$$

pour $N$ assez grand. Ceci est impossible car $B \neq 0$. L'irrationalité de $S$ est démontrée.

Remarque 1. Le lecteur familier avec la théorie élémentaire des nombres algébriques aura remarqué que le calcul de $X_{N} \widetilde{X_{N}}$ est le calcul de la norme de $X_{N}$ dans $\mathbb{Q}(\sqrt{5})$; voir [7] ou [8]. 
Remarque 2. Par le même raisonnement, il est facile de démontrer l'irrationalité de $\sum_{n=1}^{+\infty} \frac{\varepsilon^{n}}{u_{n}}$, avec $\varepsilon= \pm 1$, et où $u_{n}$ est une suite de Lucas ([6], page 41) vérifiant

$$
u_{n+1}=a u_{n}+b u_{n-1}
$$

avec $u_{0}=0, u_{1}=1, b= \pm 1, a \neq 0,|a| \geq 3$ si $b=-1$.

Remarque 3. Le lemme 1 est un cas particulier de la formule du q-binôme de Cauchy ([5], page 7).

Remerciements: L'auteur remercie le referee pour lui avoir signalé une erreur dans le première version de cet article.

\section{Bibliographie}

[1] R. André-Jeannin, Irrationalité de la somme des inverses de certaines séries récurrentes, C.R. Acad. Sci. Paris, t. 308, Sér. I, 539-541.

[2] J.M. Arnaudiès et J. Lelong-Ferrand, Cours de Mathématiques (Tome 1 : Algèbre), Dunod (3e édition) (1978).

[3] P. Bundschuh and K. Va̋âna̋nen, Arithmetical investigations of a certain infinite product, Compositio Math. 91 (1994), 175-201.

[4] M. Clayet-Michaud, Le nombre d'or, P.U.F. (Que sais-je ?) (5e édition) (1985).

[5] G. Gasper and M. Rahman, Basic hypergeometric series, Cambridge University Press (1990).

[6] P. Ribenboim, The book of prime numbers records, Springer-Verlag (1984).

[7] P. Samuel, Théorie algébrique des nombres, Hermann (1967).

[8] I.N. Stewart and D.O. Tall, Algebraic number theory, Chapman and Hall (2nd Edition) (1987).

Daniel Duverney

24 Place du Concert

F-59800 Lille 